\title{
«Das ist Demokratie»
}

\section{Interview: Bruno Kesseli}

Dr. med. et lic. phil., Chefredaktor

\author{
In der bevorstehenden Wintersession wird der Nationalrat das Tabakproduktegesetz \\ behandeln. Die Mehrheit der von Nationalrat Ignazio Cassis präsidierten Gesund- \\ heitskommission des Nationalrats (SGK-N) will das Gesetz nicht an den Bundesrat \\ zurückweisen und weicht damit von der Linie des Ständerats ab. Der ehemalige \\ Tessiner Kantonsarzt erläutert im Kurzinterview, was dies für das Gesetz bedeutet.
}

Ignazio Cassis, die Gesundheitskommission des Nationalrats (SGK-N) will das Tabakproduktegesetz im Gegensatz zum Ständerat nicht an den Bundesrat zur Überarbeitung zurückweisen. Steigen damit die Chancen, dass die Vorlage des Bundesrats durchkommt?

Es würde vor allem keine Zeit verloren: Eine Rückweisung hätte eine Verzögerung der Vorlage um rund zwei Jahre zur Folge. Wir müssen bei den Tabakprodukten eine Gesetzeslücke schliessen. Diese Erzeugnisse waren bis anhin im Lebensmittelgesetz geregelt und wurden bei dessen Revision ausgelagert. Sollte das vom Bundesrat vorgeschlagene Tabakproduktegesetz dem Parlament zu weit gehen, kann es die notwendigen Korrekturen selbst durchführen. Eine Rückweisung heisst dagegen: «Es besteht Handlungsbedarf, aber nicht so. Der Bundesrat soll nach den Vorgaben des Parlaments selber einen neuen Vorschlag bringen.» Aber dieses Gesetz ist technisch relativ einfach. Das Parlament kann die nötigen Entscheide gut selbst fällen. Zudem hat das Volk die Möglichkeit, via Referendum das letzte Wort zu haben.

Der Ständerat sprach sich gegen ein Werbeverbot für Tabakprodukte aus. Die freie Marktwirtschaft sei höher zu gewichten als die Prävention, war als Argument zu hören. Ist eine solche Argumentation nicht zynisch, wenn es um erwiesenermassen schwer gesundheitsschädigende Produkte geht?

Würde man in einem liberalen Staat alles verbieten, was der Gesundheit schadet, dann hätte man keinen liberalen Staat mehr. Freiheit - auch diejenige, sich selber zu schädigen - wird tatsächlich in vielen Weltregionen höher gewichtet als Gesundheit. Dafür haben während Jahrhunderten viele Völker gekämpft. Prävention ist heute positiv besetzt. Sie kann aber in eine Bevormundung der Menschen entgleisen, wie man aus

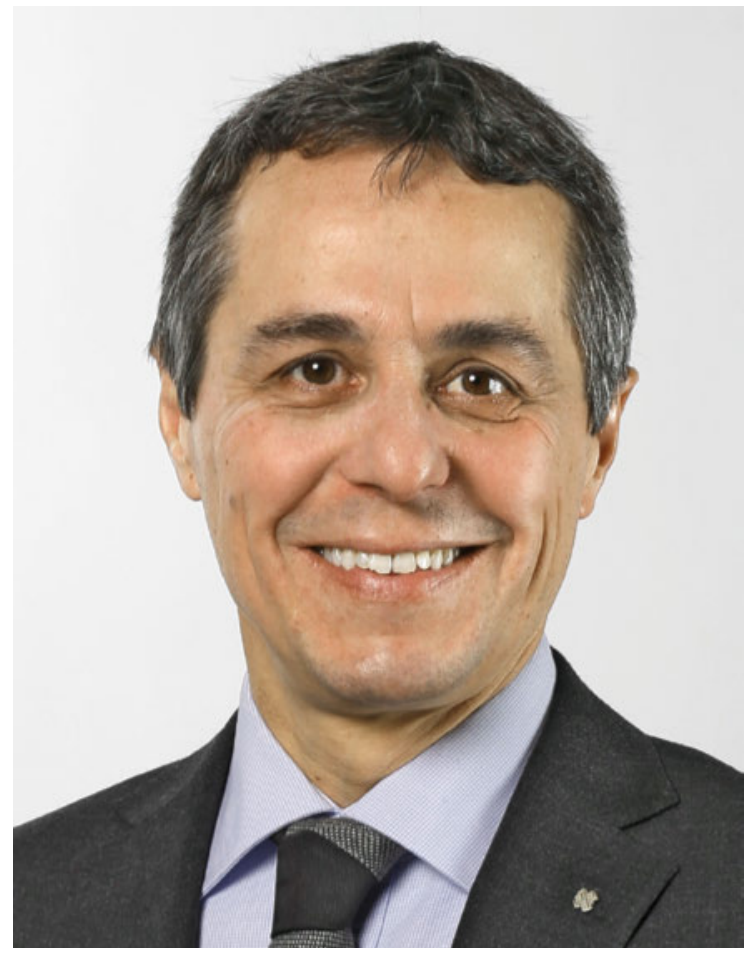

«Referendum wahrscheinlich»: Nationalrat Ignazio Cassis.

der Geschichte weiss. Bei der Drogenpolitik ist dies heute noch der Fall. Soll zum Beispiel jeder Mensch vom Staat vor sich selber geschützt werden? Wie viel Eigengefährdung, wie viel Eigenverantwortung will sich eine Gemeinschaft leisten? Wo liegt die Grenze? Das sind Fragen, die das Parlament stark beschäftigen und die von den verschiedenen Parteien unterschiedlich beantwortet werden. Das ist Demokratie.

Ist bei den herrschenden Mehrheitsverhältnissen im Parlament ein "griffiges» Tabakproduktegesetz im Sinne der Prävention und eines umfassenden 


\section{Jugendschutzes inklusive Werbeverbot überhaupt denkbar?}

Zuerst müssen wir sehen, ob der Ständerat an seinem Rückweisungsentscheid festhält. Sollte dies der Fall sein, dann wird die Vorlage mit gewissen Auflagen an den Bundesrat zurückgewiesen. Dazu zählen der Jugendschutz mit der schweizweiten Verankerung des Mindestalters 18 für den Erwerb von Tabakprodukten, die Schaffung einer rechtlichen Grundlage für Testkäufe sowie das Verbot von Werbung, die sich speziell an Minderjährige richtet. Der Ständerat will aber keine zusätzlichen Einschränkungen der Werbung, der Verkaufsförderung und des Sponsorings. Ein allgemeines Werbeverbot geht bei Erwachsenen seiner Meinung nach zu weit. Die Freiheit wurde in diesem Punkt höher gewichtet.

\section{Politik ist die Kunst des Machbaren. Was erachten Sie in der Schweiz in Bezug auf das Tabakproduktegesetz als machbar?}

Bestätigt der Nationalrat im Dezember den Entscheid seiner Kommission, das Gesetz nicht zurückzuweisen, so muss sich der Ständerat nochmals damit auseinandersetzen. Wenn die bestehende Vorlage beraten wird, gehe ich davon aus, dass die Einschränkungen der Werbung, der Verkaufsförderung und des Sponsorings im Zusammenhang mit Tabakprodukten aufgeweicht werden und dass der föderalistische Ansatz gestärkt wird. $\mathrm{Ob}$ das Gesetz am Schluss immer noch mit dem FCTC $^{* *}$ vereinbar sein wird, ist nicht sicher. Und das Volk wird nochmals das letzte Wort haben: Angesichts der heiklen Materie ist ein Referendum wahrscheinlich. Aber eben: ein Schritt nach dem anderen, ohne den Optimismus zu verlieren!

\section{Bildnachweis}

Foto zVg von Ignazio Cassis

\section{WHO-Tabakkonvention}

(Quelle: Bundesamt für Gesundheit BAG; http://www.bag.admin. ch/themen/drogen/00041/04133/14737/index.html?lang=de)

Die WHO-Tabakkonvention (Rahmenübereinkommen der WHO zur Eindämmung des Tabakgebrauchs; Framework Convention on Tobacco Control FCTC) ist der erste internationale völkerrechtliche Vertrag, der unter der Federführung der WHO ausgehandelt wurde (erster globaler Vertrag im Gesundheitsbereich). Sie enthält die Grundsätze, die weltweit für den Umgang mit Tabak und Tabakwaren gelten. Das Angebot und die Nachfrage von Tabakprodukten sollen durch verschiedene, auf wissenschaftlichen Erkenntnissen abgestützte Massnahmen zugunsten einer besseren Gesundheit weltweit reguliert werden.

Die WHO-Tabakkonvention wurde am 21. Mai 2003 von der Weltgesundheitsversammlung verabschiedet und ist seit 27. Februar 2005 in Kraft. In der Geschichte der Vereinten Nationen hat sie sich zu einem der Verträge entwickelt, dem die Staaten am raschesten und breitesten beigetreten sind.

Weltweit wurde sie von 168 Staaten unterzeichnet und von 180 Staaten ratifiziert, darunter auch von der EU (Stand: März 2016).

Die WHO-Tabakkonvention wurde erarbeitet, nachdem Krankheiten und Todesfälle im Zusammenhang mit dem Tabakkonsum weltweit stark zugenommen hatten. Es bekräftigt das Recht aller Menschen auf das für sie erreichbare Höchstmass an Gesundheit. Das FCTC stellt einen Meilenstein in der Förderung der öffentlichen Gesundheit dar und bringt eine neue rechtliche Dimension in die internationale Zusammenarbeit im Gesund heitsbereich ein.

Die WHO-Tabakkonvention verpflichtet die Vertragsparteien insbesondere zu folgenden Massnahmen:

Wirksame Massnahmen zum Schutz vor Passivrauchen am Arbeitsplatz, in geschlossenen Räumen, in öffentlichen Verkehrsmitteln, Einrichtungen und Plätzen;

Produktdeklaration und Warnhinweise auf allen Tabakprodukten Einschränkungen derTabakwerbung und desTabaksponsorings; Bekämpfung des Schmuggels, des illegalen Herstellens und der Fälschung von Tabakprodukten;

Abgabeverbot von Tabakprodukten an Minderjährige. Die Schweiz hat die WHO-Tabakkonvention am 25. Juni 2004 unterzeichnet. Damit hat der Bundesrat seinen Willen manifestiert, die WHO-Tabakkonvention auch in der Schweiz umzusetzen. Die Ratifikation ist ein Ziel des Bundesrates.

WHO Framework Convention on Tobacco Control: http://apps. who.int/iris/bitstream/10665/42811/1/9241591013.pdf?ua=1 